\title{
Regional anesthesia for a total knee arthroplasty on an adult patient with spastic diplegia and an intrathecal baclofen pump*
}

\author{
Elird Bojaxhi ${ }^{1}$, David R. Salek ${ }^{1}$, Courtney E. Sherman², Roy A. Greengrass ${ }^{1}$
}

${ }^{1}$ Department of Anesthesiology, Mayo Clinic, Jacksonville, Florida, USA

${ }^{2}$ Department of Orthopedics, Mayo Clinic, Jacksonville, Florida, USA

\begin{abstract}
We describe the clinical presentation of a patient with spastic diplegia, and its unique perioperative challenges. Opioids and antispasmodic medications are the primary therapy for managing pain and spasticity in the perioperative setting. However, such combination results in several side-effects and their sedative properties are synergistic. A 64-year-old woman with a history of spastic diplegia and an intrathecal baclofen pump for the treatment of her lower extremity spasticity was scheduled for a third elective left knee arthroplasty. She requested a regional anesthetic for the anticipated surgery and an opioid sparing postoperative analgesic regiment. We describe the successful use of a lumbar plexus and a sciatic nerve block as the primary anesthetic for the surgery and the use of a continuous lumbar plexus catheter for the postoperative course. Based on our patient's past anesthetic history, a regional anesthetic/analgesic technique is the ideal strategy in controlling perioperative pain and spasticity
\end{abstract}

Keywords: cerebral palsy; intrathecal baclofen pump; regional anesthesia; spastic diplegia

\section{Introduction}

Cerebral palsy $(\mathrm{CP})$ is a term used to describe a heterogeneous group of disorders that are characterized by nonprogressive neurologic dysfunction resulting from any event causing cerebral injury, usually occurring early in development [1]. Spastic diplegia (SD) is a type of CP described in roughly $22 \%$ of all $\mathrm{CP}$ cases [1], presenting with spasticity and often painful contractures which ultimately result in muscle and

Address for correspondence: Elird Bojaxhi, MD

Department of Anesthesiology

Mayo Clinic

4500 San Pablo Road

Jacksonville, FL 32224, USA

E-mail: bojaxhi.elird@mayo.edu joint breakdown requiring surgical corrections over time. Patients with SD are prone to poor postoperative pain control, which can manifest as increased perioperative morbidity [2].

We present a patient with SD and an intrathecal baclofen pump anticipating a third left total knee arthroplasty (TKA). A combined lumbar plexus and sciatic nerve block was successfully used to provide surgical anesthesia for TKA and a continuous lumbar plexus catheter provided postoperative analgesia.

\section{Case report}

A 64-year-old woman $(74 \mathrm{~kg})$ with a history of SD presented for a third left knee arthroplasty and quadriceps tendon reconstruction. Her medical history also included depression, anxiety, and chronic low back pain. On preoperative evaluation, she reported her knee

\footnotetext{
* Presentation: $41^{\text {st }}$ Annual (ASRA) Regional Anesthesiology and Acute Pain Medicine Meeting, March 31-April 2, 2016, New Orleans,
} LA, Abstract: \#1378, Medically Challenging Cases. C2017 Mayo Foundation for Medical Education and Research 
pain at $4 / 10$ at rest and $8 / 10$ with ambulation on the 11-point Numeric Rating Scale (NRS-11) ranging from 0 (no pain) to 10 (worst pain). The patient was taking $7.5 \mathrm{mg}$ of hydrocodone four times per day as needed for pain, and had an intrathecal baclofen pump for lower extremity spasticity.

From two prior general anesthetics, the patient reported poor pain control due to spasticity and hypertonicity, re-intubation for respiratory depression in the recovery room, several opioid-related side effects (nausea, vomiting, sedation, and confusion), and difficulty participating in physical therapy.

The patient's symptoms of SD were originally noted in childhood as she had difficulty learning to walk. Her condition primarily involved spasticity and hypertonicity of her lower extremities which caused flexion and decreased range of motion of the hips and knees. The spasticity resulted in difficulty in straightening the legs, and she used crutches or a walker to facilitate ambulation and support her gait. However, with development of osteoarthritis of her left knee and two prior failed knee surgeries, ambulation was further burdened by pain. On examination, the patient had an antalgic gait with an inability to straighten her foot or knee. Strength of the lower extremities was intact, but numbness of the skin over the left knee and lower thigh was noted.

Upon reviewing the procedure report of the intrathecal baclofen pump placement, the catheter entered the dural sac at the L5-S1 interspace and threaded cephalad to L1-L2. The catheter was then tunneled under the skin to the pump located above her right hip which was also noted on examination.

The surgical procedure was anticipated to last 2-3 hours; the incision involved the lower quadriceps muscle for the tendon reconstruction, and utilized a tourniquet placed on the upper thigh. Since a neuraxial technique, such as a spinal, could damage the intrathecal catheter which was located midline at the appropriate intervertebral levels, the proposed anesthetic plan was a lumbar plexus block with catheter placement, a single injection sciatic nerve block, and intravenous sedation during the surgery. While taking the history of SD into consideration, the risks and benefits of a peripheral nerve block and use of a peripheral nerve catheter were discussed with the patient.

A posterior approach to the left lumbar plexus block was performed as described by Winnie et al. [3], but under nerve stimulator guidance and not paresthesia. The patient was placed in the right lateral position with her left hip and knee flexed (Sims' position). Under aseptic technique, an $18 \mathrm{G}$ stimulating Touhy needle was inserted at the perpendicular intersection of an intercristal line at the iliac crests (corresponding to L4-L5), with a second line running through the posterior superior iliac spine and parallel to the spine. A quadriceps twitch was elicited via nerve stimulator at $0.55 \mathrm{~mA}$, and 20 cc of $0.5 \%$ ropivacaine was incrementally injected after negative aspiration of blood or cerebral spinal fluid. A $20 \mathrm{G}$ soft tip catheter was then advanced through the needle, with $3 \mathrm{~cm}$ left beyond the tip of the needle.

The sciatic nerve was blocked while the patient was still in the right Sims' position using the posterior Labat approach [4]. A line was drawn from the posterior superior iliac spine to the greater trochanter. At the midpoint, a perpendicular line running caudate was drawn measuring $4 \mathrm{~cm}$, marking the needle insertion site. Under aseptic technique, a 20 G B.Braun stimulating needle (Braun Medical Inc, Bethlehem, PA) was advanced and elicited a plantar flexion of the foot at $0.46 \mathrm{~mA}$. After negative aspiration, $10 \mathrm{cc}$ of $0.5 \%$ ropivacaine was incrementally injected through the needle.

The patient tolerated the procedure well, and no complications were noted. Approximately 20 minutes after the completion of the block, thermal anesthesia to ice was noted circumferentially around the left thigh and knee. Marked weakness was also noted of the flexion of the left hip, extension of the knee, and plantar flexion of the foot.

In the operating room, the patient received propofol IV sedation while spontaneously ventilating with supplemental oxygen via nasal cannula. The surgical procedure was uneventful, and the patient was comfortable. Sedation was discontinued in the operating room at the conclusion of the procedure, and the patient was awake prior to arrival in the Post Anesthesia Care Unit (PACU). During her 73 minute stay in the PACU, the patient reported her pain at $0 / 10$ on a NRS-11 at rest, with thermal anesthesia to ice still present circumferentially around the left thigh. Opioid analgesia was not needed intraoperatively or in the PACU, and the patient reported no nausea. Before leaving the PACU, the patient's lumbar plexus catheter was connected to an electronic infusion pump running $0.2 \%$ ropivacaine at $10 \mathrm{cc} / \mathrm{hr}$ with a patient-controlled bolus of $8 \mathrm{cc}$ every 60 minutes. Postoperative analgesic regimen consisted of $1000 \mathrm{mg}$ of acetaminophen taken orally every 6 hours and $2 \mathrm{mg}$ of hydromorphone taken orally every 4 hours as needed for pain. By postoperative day (POD) 3, the patient was transitioned off oral hydromorphone and back onto her preoperative regimen of hydrocodone and acetaminophen.

As is routine at our institution, the left lumbar plexus catheter was followed daily to assess block function, patient's pain control, and any complications. While the peripheral catheter was in place, the patient demonstrated decreased sensation over the left anterior thigh and mild weakness of the quadriceps muscle. The peripheral nerve catheter was discontinued on the evening of POD 2, and complete resolution of the block was noted on POD 3. 
The first dose of opioid was requested in the evening of POD 0 (approximately 12 hours after the placement of the nerve blocks), and the patient reported the pain to be $3 / 10$ on a NRS-11. Figure 1 summarizes the patient's opioid use (in mg of oral morphine equivalences) and the mean and maximum pain levels during the patient's hospital stay. Pain scores were as per routine nursing assessment, and maximum pain scores were related to activity and physical therapy. During her postoperative course, the patient did not experience any episodes of nausea or vomiting, nor did she feel overly sedated.

\section{Discussion}

There are many inherent challenges in treating patients with $\mathrm{CP}$ which vary greatly by neurologic impairment and degree of disability. Our patient's disability, due to SD, resulted in spasticity and pain of her lower extremities which caused poor postoperative pain control with prior general anesthetics. Strategies to reduce spasticity and exacerbation of postoperative pain include administration of diazepam or baclofen [5]. However, such strategies in adjunct to a general anesthetic and opioid analgesia also place these patients at increased risk for prolonged emergence, respiratory depression, and excessive sedation.

Epidural analgesia to reduce pain and lower extremity spasticity has been shown to be beneficial in pediatric patients with $\mathrm{CP}$ undergoing orthopedic procedures [5]. However, the presence of an intrathecal catheter presents unique challenges when considering neuroaxial anesthetic or analgesic techniques. A case report described the uneventful use of an epidural catheter for labor analgesia on a patient with an intrathecal morphine pump [6]. However, the lack of clinical evidence leads many clinicians to consider the presence of a spinal cord stimulator or intrathecal pump as a relative contraindication to the performance of spinal or epidural anesthesia. In our case report, the location of the intrathecal catheter was described, based on recent radiological studies, as entering the dural sac at L5-S1; therefore, a spinal needle could have punctured or damaged the catheter. Placement of an epidural catheter may also interfere with the intrathecal catheter by kinking, dislodging, or introducing a source of infection.

For most primary TKA in the general population, current clinical practice often includes a femoral nerve block [7] or a saphenous nerve block [8], either via a single injection or continuous infusion, to provide analgesia to the anterior component of the knee. The sciatic nerve also plays a role in the innervation of the posterior knee; however, the evidence is not yet conclusive in blocking the sciatic nerve [9], and the block is not routinely performed for postoperative analgesia. As described in our case report, the patient was undergoing a second revision of her TKA, the incision further extended to the thigh, and a tourniquet was applied on the upper thigh. In an effort to provide surgical anesthesia via a peripheral block to include the femoral, femoral cutaneous, obturator, and sciatic nerve, we chose a combined lumbar plexus and sciatic block technique for the case. Luber et al. [10] previously described this technique for providing primary anesthesia during a TKA and for postoperative analgesia as continuous catheters were used in both blocks.

Considering the patient's underlying bilateral lower extremity weakness and spasticity, we decided not to place a continuous sciatic block to avoid extensive weakness of the operative leg. Overall, the patient was very satisfied with her analgesic regimen, and reported

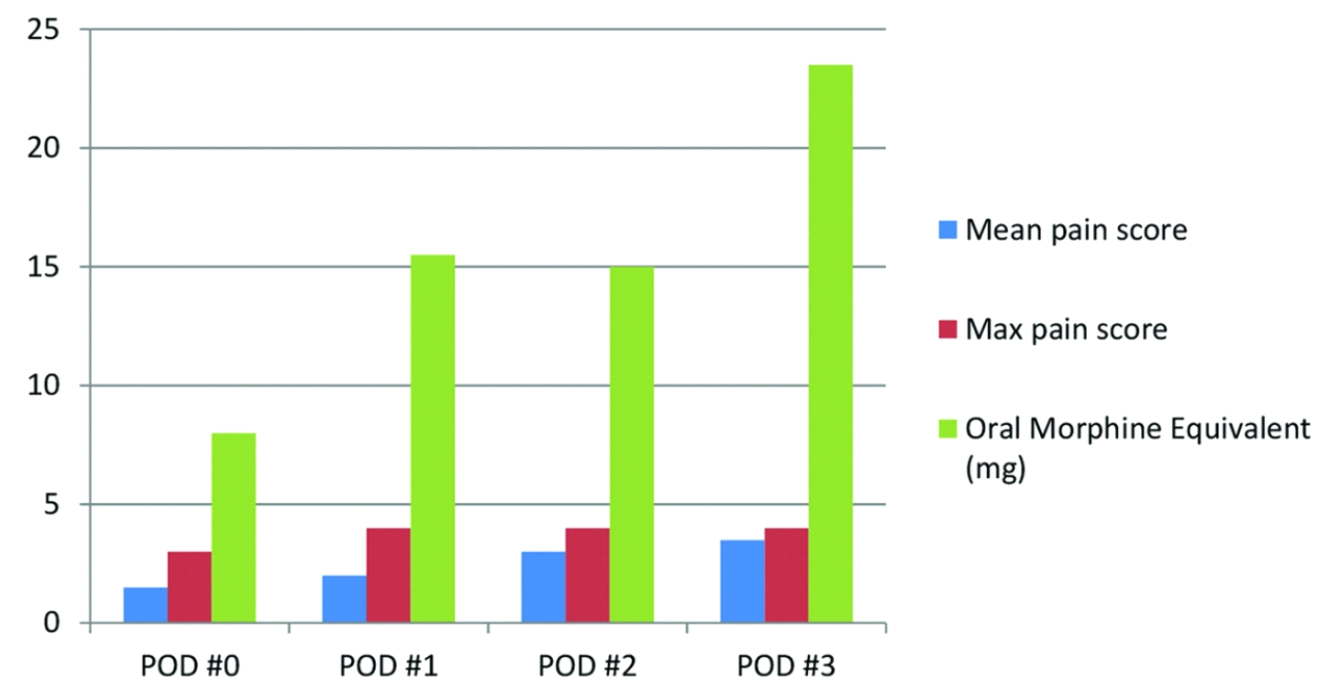

Fig. 1. The mean and maximum pain scores, via the NRS-11, are plotted against oral morphine equivalent doses. Note the peripheral nerve catheter was removed in the evening of POD 2. POD: postoperative day 
improved participation with ambulation and physical therapy when compared to her previous knee surgeries under general anesthesia.

To our knowledge, there have been no specific studies that have addressed nerve injury due to a regional anesthetic in patients with $\mathrm{CP}$. Due to the relative paucity of cases, there are no established Clinical Practice Guidelines for these patients. Because the pathophysiology of $\mathrm{CP}$ is described as a nonprogressive neurological dysfunction due to damage of the premature brain in early pregnancy [5], the risk of any peripheral nerve damage in this patient population secondary to peripheral nerve blockade might be that of the general population. Unfortunately, many patients with CP suffer from cognitive impairment, presenting a barrier for a thorough evaluation, and inadequate pain control or potential complications due to a peripheral nerve block may go unnoted. As such, the primary strength of this report is that our patient was cognitively intact and could easily self-report symptoms. The primary limitation of this report is that it is a single case in a disease that has many phenotypic variations.

\section{Conclusions}

We present a case of a patient with SD and an intrathecal baclofen pump who presented for a third TKA with quadriceps tendon repair. As her prior anesthetic history was significant for poor postoperative pain control and intolerance to opioids, a lumbar plexus and a sciatic nerve block was utilized to provide anesthesia intraoperatively, and a lumbar plexus catheter for postoperatively analgesia. Our patient reported a great degree of satisfaction due to her pain being well-controlled with minimal use of opioids, helped with participation in physical therapy, and spared opioidrelated side effects such as nausea, vomiting, and sedation. As discussed, a patient with SD and an intrathecal baclofen pump presents unique challenges for the anesthetic and analgesic plan. However, these challenges do not preclude the option for anesthesia under peripheral nerve blocks, and we would encourage for a regional anesthetic plan to part of the discussion between the patient and the anesthesiologist.

\section{References}

1. Mutch L, Alberman E, Hagberg B, Kodama K, Perat MV. Cerebral palsy epidemiology: where are we now and where are we going? Dev Med Child Neurol 1992; 34: 547-551. DOI: 10.1111/j.14698749.1992.tb1 1479.x

2. Hirsh AT, Kratz AL, Engel JM, Jensen MP. Survey results of pain treatments in adults with cerebral palsy. Am J Phys Med Rehabil 2011; 90: 207-216. DOI: 10.1097/ PHM.0b013e3182063bc9

3. Winnie AP, Ramamurthy S, Durrani Z, Radonjic R. Plexus blocks for lower extremity surgery: new answers to old problems. Anesthesiol Rev 1974; 1: 11-16

4. Taboada M, Rodríguez J, Alvarez J, Cortés J, Gude F, Atanassoff PG. Sciatic nerve block via posterior Labat approach is more efficient than lateral popliteal approach using a double-injection technique: a prospective, randomized comparison. Anesthesiology 2004; 101: 138-142

5. Nolan J, Chalkiadis GA, Low J, Olesch CA, Brown TC. Anaesthesia and pain management in cerebral palsy. Anaesthesia 2000; 55: 32-41. DOI: 10.1046/j.1365-2044.2000.01065.x

6. Tarshis J, Zuckerman JE, Katz NP, Segal S, Mushlin PS. Labour pain management in a parturient with an implanted intrathecal pump. Can J Anaesth 1997; 44: 1278-1281. DOI: 10.1007/ BF03012776

7. Paul JE, Arya A, Hurlburt L, Cheng J, Thabane L, Tidy A, et al. Femoral nerve block improves analgesia outcomes after total knee arthroplasty: a meta-analysis of randomized controlled trials. Anesthesiology 2010; 113: 1144-1162. DOI: 10.1097/ ALN.0b013e3181f4b18

8. Moore DM, O'Gara A, Duggan M. Continuous saphenous nerve block for total knee arthroplasty: when and how? Reg Anesth Pain Med 2013; 38: 370-371. DOI: 10.1097/ AAP.0b013e318294fe4b

9. Abdallah FW, Brull R. Is sciatic nerve block advantageous when combined with femoral nerve block for postoperative analgesia following total knee arthroplasty? A systematic review. Reg Anesth Pain Med 2011; 36: 493-498. DOI: 10.1097/ AAP.0b013e318228d5d4

10. Luber MJ, Greengrass R, Vail TP. Patient satisfaction and effectiveness of lumbar plexus and sciatic nerve block for total knee arthroplasty. J Arthroplasty 2001; 16: 17-21. DOI: 10.1054/arth.2001.16488

\section{Conflict of interest}

Nothing to declare 